Tonini, IGO, Vaz, DSS \& Mazur CE (2020). Gut-brain axis: relationship between intestinal microbiota and mental disorders. Research, Society and Development, 9(7):1-14, e499974303.

\title{
Eixo intestino-cérebro: relação entre a microbiota intestinal e desordens mentais
}

Gut-brain axis: relationship between intestinal microbiota and mental disorders

Eje cerebral intestino: relación entre microbiotes intestinales y trastornos mentales

Recebido: 07/05/2020 | Revisado: 14/05/2020 | Aceito: 14/05/2020 | Publicado: 23/05/2020

Ingrid Gabriela de Oliveira Tonini

ORCID: https://orcid.org/0000-0003-2568-8721

Centro Universitário Campo Real. Guarapuava, Paraná, Brasil.

E-mail: ingrid.tonini@hotmail.com

Diana Souza Santos Vaz

ORCID: https://orcid.org/0000-0001-5969-8122

Universidade Estadual de Campinas. Campinas, São Paulo, Brasil

E-mail: nutridianavaz@gmail.com

Caryna Eurich Mazur

ORCID: https://orcid.org/0000-0002-1278-5963

Universidade Estadual do Centro-Oeste Guarapuava, Paraná, Brasil.

E-mail: carynanutricionista@gmail.com

\section{Resumo}

O termo "eixo intestino-cérebro" tem sido amplamente utilizado no estudo da comunicação entre o trato gastrointestinal e o sistema nervoso central, uma relação vital para a manutenção da homeostase e cujo desequilíbrio pode resultar em alterações na resposta ao estresse e no comportamento humano. Esta revisão visa apresentar e discutir os achados de estudos sobre o eixo intestino-cérebro e a possível relação entre disbiose intestinal e desordens mentais. A pergunta norteadora foi: "Como a saúde intestinal pode afetar o cérebro e seu funcionamento?". De cinquenta artigos encontrados em todas as bases de dados, foram selecionados sete para integrar esta revisão, sendo todos publicados em inglês. Os achados de todos os artigos discutidos nesta revisão demonstram impacto direto e significativo da microbiota intestinal sobre o sistema nervoso central, observando-se comportamentos relacionados à ansiedade, depressão e estresse frente à disbiose.

Palavras-chave: Ansiedade; Depressão; Microbioma gastrointestinal; Transtornos mentais. 


\begin{abstract}
The term "gut-brain axis" has been widely used in the study of communication between the gastrointestinal tract and the central nervous system, a vital relationship for the maintenance of homeostasis and whose imbalance can result in changes in the response to stress and in behavior. This review aims to present and discuss the findings of studies about the gut-brain axis and the possible relationship between intestinal dysbiosis and mental disorders. The guiding question was: "How can intestinal health affect the brain and its functioning?" Of fifty articles found, seven were selected to integrate this review, all published in English. The findings of all articles discussed in this review demonstrate a direct and significant impact of the intestinal microbiota on the central nervous system, observing behaviors related to anxiety, depression and stress in the face of changes in the microbiota.
\end{abstract}

Key words: Anxiety; Depression; Gastrointestinal microbiome; Mental disorders.

\title{
Resumen
}

El término "eje intestino-cerebro" se ha utilizado ampliamente en el estudio de la comunicación entre el tracto gastrointestinal y el sistema nervioso central, una relación vital para el mantenimiento de la homeostasis y cuyo desequilibrio puede provocar cambios en la respuesta al estrés y al comportamiento en general. Esta revisión tiene como objetivo presentar y discutir los hallazgos de los estudios sobre el eje intestino-cerebro y la posible relación entre la disbiosis intestinal y los trastornos mentales. La pregunta guía fue: "¿Cómo puede afectar la salud intestinal al cerebro y su funcionamiento?" De cincuenta artículos encontrados, se seleccionaron siete para integrar esta revisión, todos publicados en inglés. Los hallazgos de todos los artículos discutidos en esta revisión demuestran un impacto directo y significativo de la microbiota intestinal en el sistema nervioso central, observando comportamientos relacionados con la ansiedad, la depresión y el estrés frente a los cambios en la microbiota.

Palabras clave: Ansiedade; Depresión; Microbioma gastrointestinal; Trastornos mentales.

\section{Introdução}

Nos últimos anos, o número de pessoas com algum diagnóstico clínico de transtorno mental tem aumentado de maneira substancial, sendo que, dentre esses problemas, a depressão é o mais frequente e incapacitante, afetando cerca de 300 milhões de pessoas de 
todas as idades no mundo (OPAS/OMS, 2018). Já a nível nacional, o Brasil ocupa o quinto lugar na lista dos países com maior prevalência desse transtorno (OMS, 2017).

Ainda, de acordo com levantamento da Organização Mundial da Saúde (OMS), em 2017, o Brasil era o país com o maior número de pessoas afetadas por transtorno de ansiedade, problema que atingia 9,3\% da população (OMS, 2017). Ressalta-se que, quando mais intensa, a ansiedade também pode se tornar incapacitante, imobilizando o indivíduo e impedindo-o de reagir a determinadas situações cotidianas (Ministério da Saúde, 2015).

Do ponto de vista neurobiológico, a depressão associa-se com um desequilíbrio nos sistemas endócrino, imune, metabólico e nervoso, com enfoque na capacidade neurotransmissora, desequilíbrio este que, segundo estudos envolvendo animais, pode ser modulado pelos microrganismos presentes na microbiota intestinal (Kelly, Clarke, Cryan \& Dinan, 2016). Isto se deve, entre outros fatores, à capacidade das bactérias intestinais em clivar e metabolizar determinados nutrientes, além de exercer um papel determinante na síntese de aminoácidos, como o ácido gama-aminobutírico (GABA) e o triptofano, além de monoaminas, como a serotonina, a histamina e a dopamina, neurotransmissores que chegam ao sistema nervoso central por meio da corrente sanguínea e dos neurônios presentes no sistema nervoso entérico (Stilling, Dinan \& Cryan, 2013).

Sabe-se que desordens emocionais podem possuir etiologias multifatoriais; no entanto, cada vez mais a literatura tem demonstrado a importância da relação entre o intestino e o cérebro nesses transtornos.

Ainda no embrião, cérebro e sistema digestivo estão intimamente relacionados, compartilhando terminações nervosas e, deste modo, se comunicando amplamente via sinapse e neurotransmissores (Ozan, Tanik \& Inan, 2019). Consequentemente, o funcionamento de um órgão influencia diretamente no outro, e, assim, prejuízos na função intestinal podem acarretar em desordens no sistema nervoso central (Del'Arco, Magalhães \& Quilici, 2017).

O termo "eixo intestino-cérebro" tem sido amplamente utilizado no estudo da comunicação bidirecional entre o trato gastrointestinal e o sistema nervoso central, um sistema vital para a manutenção da homeostase e cujo desequilíbrio pode resultar em alterações na resposta ao estresse e comportamento no geral (Cryan \& O’Mahony, 2011).

Diante do exposto, o objetivo desta revisão bibliográfica é apresentar e discutir os achados de estudos sobre o eixo intestino-cérebro e a possível relação entre disbiose intestinal e desordens mentais, tais como a depressão e o transtorno de ansiedade. 


\section{Metodologia}

Uma pesquisa é realizada com o objetivo e trazer novos saberes como preconiza Pereira et al. (2018). O presente estudo trata-se de uma revisão integrativa da literatura. A pergunta norteadora foi: "Como a saúde intestinal pode afetar o cérebro e seu funcionamento?". Para a busca dos artigos, foram utilizadas as bases de dados Scielo, Science Direct, PubMed, Lilacs e Medline, empregando-se os seguintes termos: "intestino", "cérebro", "microbiota intestinal" e "transtornos mentais", em língua portuguesa e inglesa.

Os critérios de seleção estabelecidos foram: artigos originais, realizados com seres humanos e animais, publicados tanto em português quanto em inglês, durante o período de 2010 a 2020, disponíveis eletronicamente em formato completo, e cujo tema se relaciona ao eixo intestino-cérebro (Figura 1).

Figura 1. Fluxograma das etapas de busca e seleção dos artigos.

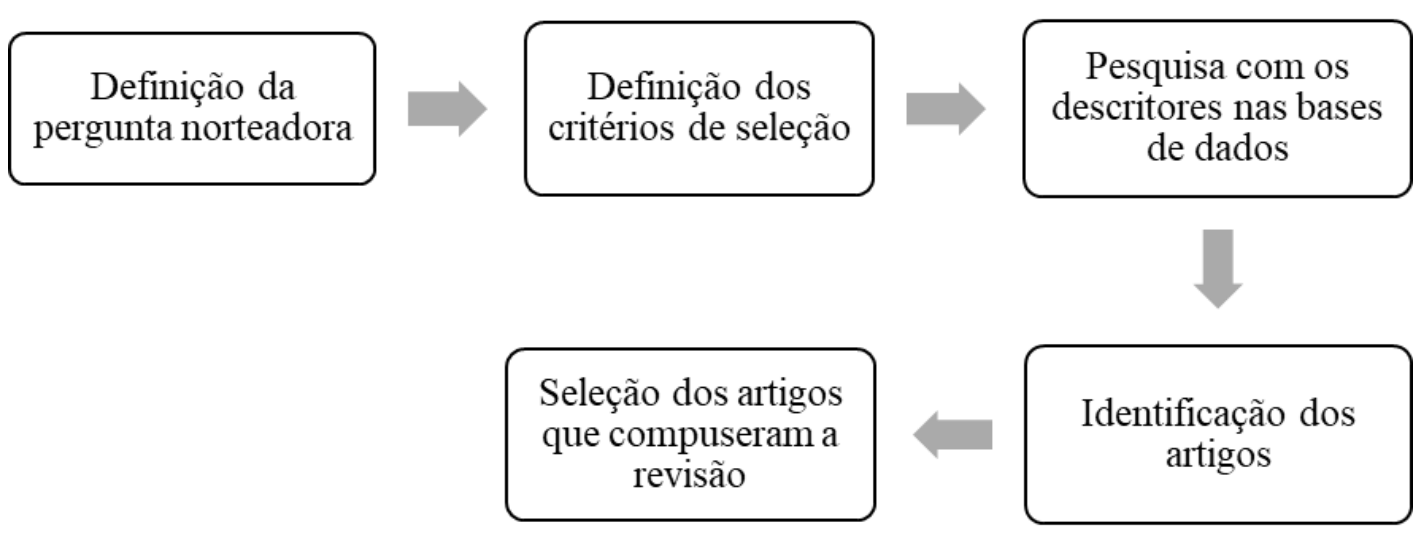

Fonte: Autores.

Neste contexto, a presente revisão bibliográfica propõe-se a apresentar e discutir os resultados encontrados nos estudos selecionados.

\section{Resultados e Discussão}

Foram encontrados, em todas as bases de dados utilizadas, um total de 50 artigos com tema relacionado ao eixo intestino-cérebro. Destes, porém, apenas 7 foram selecionados para integrar esta revisão, levando-se em consideração os critérios de seleção pré-estabelecidos. Ressalta-se a escassez de estudos originais na área pesquisada, especialmente envolvendo 
seres humanos, e, diante deste fato, justifica-se o baixo número de artigos analisados nesta revisão. Todos os estudos selecionados foram publicados em língua inglesa, e apenas um deles foi realizado com seres humanos, sendo todos os outros com ratos.

\subsection{Constituição e importância da microbiota intestinal}

A microbiota intestinal é constituída majoritariamente por bactérias, mas também contém arqueias, protozoários, fungos e vírus (Dinan et al., 2015). Neste sistema, os microrganismos comensais e os seres humanos estabelecem uma relação mútua simbiótica e harmônica, a qual envolve proteção contra patógenos, digestão e absorção de nutrientes e síntese de vitaminas, além da metabolização de substâncias tóxicas por parte dos comensais, e o fornecimento de condições ideais para sobrevivência destes por parte do hospedeiro (Lach et al., 2017; Dinan et al., 2015).

No entanto, essa relação ultrapassa as funções previamente citadas, visto que as bactérias intestinais podem regular o desenvolvimento, a função e o comportamento cerebral, por meio das vias de sinalização imunológica, endócrina, metabólica e neural (Liu et al., 2019), bem como sintetizar grande parte dos neuroquímicos essenciais às funções orgânicas, por exemplo o sistema serotoninérgico, que desempenha um papel fundamental na regulação da atividade emocional, todavia, não é adequadamente desenvolvido na ausência ou disfunção da microbiota (Clarke et al., 2013).

Diante de tais evidências, é bem estabelecido o papel da microbiota intestinal sobre o comportamento e as emoções. Isto porque, além de sintetizar uma grande variedade de neurotransmissores, ela é capaz de reduzir quadros de inflamação de baixo grau (Dinan, Stanton \& Cryan, 2013; Kelly et al., 2015), considerando-se que a depressão está associada à presença de biomarcadores inflamatórios, como a Interleucina-6 (IL-6), fator de necrose tumoral alfa (TNF- $\alpha$ ) e proteína C-reativa (PCR), os quais também podem estar presentes em estados de ansiedade (Kelly et al., 2015; Dinan \& Cryan, 2017).

Tendo em vista a relevância da microbiota sobre a atividade cerebral, a disbiose intestinal pode implicar em sérias consequências sobre o funcionamento do sistema nervoso, tanto da perspectiva neurobiológica quanto da saúde mental (Dinan \& Cryan, Ibidem, 2017), podendo acarretar, deste modo, em transtornos como a depressão e a ansiedade.

A Figura 2 adaptada de Bastiaanssen et al. (2020), mostra o impacto da microbiota intestinal na regulação do eixo hipotálamo-hipófise-adrenal (HPA). Como ilustrado, alterações na microbiota causam uma hiperativação do eixo HPA, seguida por interrupção dos 
(CC BY 4.0) | ISSN 2525-3409 | DOI: http://dx.doi.org/10.33448/rsd-v9i7.4303

circuitos neurais e dos níveis de neurotransmissores, produção excessiva de citocinas próinflamatórias pelo sistema imunológico e ruptura da barreira intestinal (Bastiaanssen et al., 2020).

Figura 2. Impacto da microbiota intestinal no eixo intestino-cérebro na saúde e nas desordens mentais.

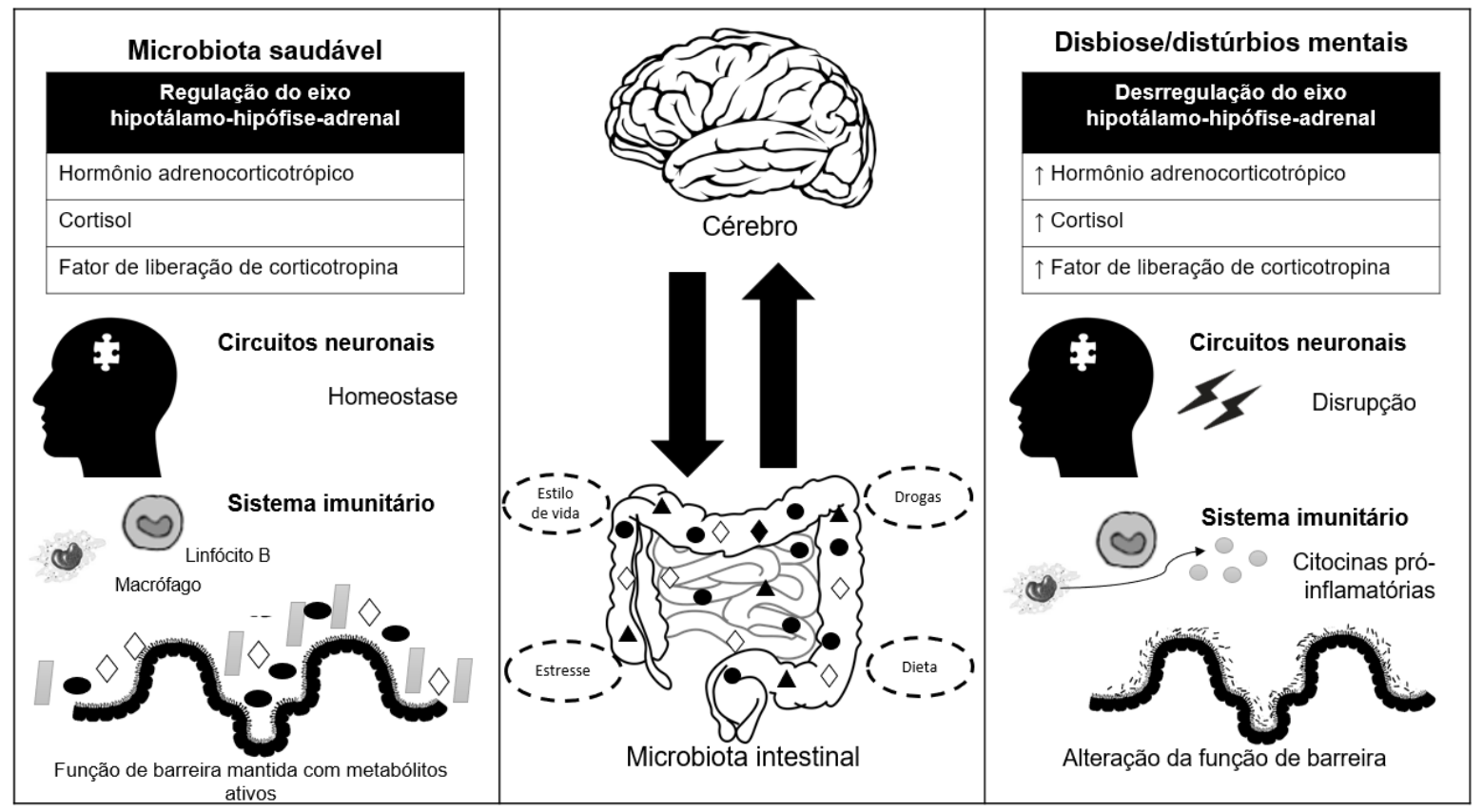

Adaptado de Bastiaanssen et al. (2020).

Com base nisso, os moduladores da microbiota, como os probióticos e os prebióticos, têm sido amplamente relacionados à atenuação dos sintomas de ansiedade, depressão e estresse, fato que se deve à sua capacidade de modular a composição da microbiota intestinal, além de reduzir os níveis de cortisol, promovendo, assim, o bem estar (Lach et al., 2017; Gareau et al., 2011; Schmidt et al., 2015).

\subsection{Microbiota intestinal e as desordens mentais: papel do eixo intestino-cérebro}

Zhang et al. (2019) investigaram o papel da microbiota intestinal no delirium pósoperatório (DPO), uma complicação neurológica ocasionada por processos cirúrgicos e pela anestesia. Sua etiologia não está totalmente esclarecida na literatura, mas há a teoria de que, um estado inflamatório pré-existente, quando exposto ao estímulo nocivo da cirurgia, pode desencadear uma cascata de citocinas e mediadores inflamatórios, resultando em lesão neuronal (Cerejeira, Firmino \& Vaz-Serra, 2010). Suas manifestações clínicas incluem 
desorientação, dificuldade na fala e prejuízo na memória, além de distúrbios emocionais, como ansiedade, medo, irritabilidade e depressão (Barbosa et al., 2008).

No estudo em questão, 71 ratos machos foram divididos aleatoriamente em dois grupos, um controle e outro que seria submetido a anestesia e cirurgia - uma laparotomia simples. Para a análise da composição microbiológica intestinal, realizou-se coleta de amostras fecais e posterior sequenciamento genético destas. Os resultados do estudo demonstraram que uma composição anormal da microbiota intestinal contribuiu para a patogênese do delirium pós-operatório. O estudo de Zhang et al. (2019) é o primeiro a relatar a associação entre a microbiota intestinal e o DPO, e, devido à escassez de pesquisas com esta temática, os mecanismos dessa relação ainda não estão elucidados.

O estudo de Pan et al. (2019) buscou determinar se uma expressão genética e um perfil comportamental alterados poderiam ser revertidos pela recuperação da microbiota intestinal em camundongos livres de microrganismos. Para isto, mensurou-se a expressão de genes relacionados a neurotransmissores monoamínicos, como a serotonina e a dopamina, no hipocampo de camundongos livres de microrganismos e de camundongos livres de patógenos específicos, a fim de explorar o efeito da microbiota intestinal no funcionamento desses sistemas.

Como resultado, os pesquisadores observaram comportamento ansiolítico nos ratos com intestino estéril, em comparação com os ratos livres apenas de patógenos específicos, embora a colonização intestinal daqueles animais não tenha sido suficiente para reverter sua expressão gênica alterada. De modo semelhante, outro estudo (Clarke et al., 2013) demonstrou que a ansiedade presente em animais livres de microrganismos foi normalizada após a restauração da microbiota intestinal.

Sabe-se que as células enterocromafins (CE) do intestino são responsáveis pela síntese de, aproximadamente, $95 \%$ de toda a serotonina corporal (Gershon \& Tack, 2007), e já se sabe que a microbiota intestinal exerce um importante papel nesse sistema (Reigstad et al., 2015). Neste contexto, Yano et al. (2015) observaram que os metabólitos produzidos por bactérias formadoras de esporos aumentam os níveis de 5-HT nas CEs, embora os mecanismos desta ação ainda precisem ser esclarecidos.

Liu et al. (2020) buscaram determinar o efeito da administração de diversas cepas de probióticos na regulação da microbiota intestinal, bem como seu impacto em sintomas depressivos em ratos sob condições de estresse. As formulações probióticas continham Lactobacillus plantarum, L. rhamnosus, Bifidobacterium lactis, B. breve e Pediococcus pentosaceus, sendo administradas via oral, diariamente, durante oito semanas. Após o 
tratamento, os animais foram submetidos a testes comportamentais. Os autores observaram mudanças na composição da microbiota intestinal dos ratos devido às condições de estresse, havendo, porém, a restauração dessa composição com a administração dos probióticos. Além disso, após o tratamento, observou-se redução significativa dos níveis de corticosterona sérica entre os animais, bem como modulação dos comportamentos relacionados ao estresse, o que faz dos probióticos uma ferramenta útil para amenizar os sintomas dessa condição (Liu et al., Ibidem, 2020).

Outro estudo analisado nesta revisão foi o de Xiao et al. (2018), que investigaram o efeito da microbiota intestinal sobre a ansiedade induzida pela abstinência de álcool. O estudo foi realizado com roedores, os quais foram submetidos à ingestão forçada e progressiva de solução alcoólica, por meio de gavagem, além da adição dessa solução a 5\% em sua água potável. Na primeira semana, a concentração da solução foi de $5 \%$, já na segunda semana, foi de $10 \%$, na terceira, $20 \%$, e na quarta, $35 \%$.

Os pesquisadores observaram que o consumo alcoólico não alterou o número de espécies de bactérias entéricas, contudo, a abundância de Lactobacillus foi reduzida, ou seja, houve alteração na composição da microbiota, fato que contribuiu para o desenvolvimento da ansiedade induzida pela abstinência alcoólica, segundo os autores do estudo. Além disso, foi realizado um transplante fecal dos animais expostos ao álcool para ratos de um grupo controle, o que acarretou em comportamentos depressivos nestes últimos, provavelmente devido à alteração da composição de suas bactérias intestinais. Portanto, o estudo conclui que a microbiota intestinal pode ser uma importante ferramenta terapêtica para atenuar os sintomas da abstinência alcoólica.

Kelly et al. (2016) também avaliaram se ratos que receberam transplante da microbiota de indivíduos depressivos apresentariam um fenótipo igualmente depressivo. A amostra foi composta por trinta e quatro pacientes, recrutados de ambulatórios e clínicas psiquiátricas, além de vinte e oito ratos machos. Primeiramente, os animais receberam tratamento com antibiótico durante vinte e oito dias, e, após este período, receberam a microbiota dos doadores via sonda oral. A microbiota foi coletada de amostras fecais dos três pacientes mais gravemente deprimidos e de três pacientes-controle saudáveis. Foi possível observar que os ratos que receberam o transplante da microbiota dos pacientes deprimidos demonstraram comportamentos relacionados à anedonia (perda/ausência da capacidade de sentir prazer) e ansiedade, corroborando com o estudo anterior (Xiao et al., 2018), que também verificou relação entre o desequilíbrio da microbiota intestinal e depressão. 
Assim, o estudo conclui que é possível reproduzir comportamentos depressivos em ratos que recebem transplante da microbiota intestinal de doadores com depressão. Com este achado, abre-se espaço para novas pesquisas que avaliem se o contrário também é verdadeiro, isto é, se o transplante de uma microbiota saudável pode amenizar sintomas depressivos em indivíduos com este transtorno.

No estudo de Burokas et al. (2017), investigou-se o efeito do tratamento prebiótico crônico sobre o comportamento ligado à ansiedade, depressão e estresse em ratos. Para isto, foram administrados prebióticos dos tipos frutooligossacarídeo (FOS) e galactoligossacarídeo (GOS), tanto isolados quanto combinados entre si.

Como resultado, observou-se que o uso dos prebióticos modificou acentuadamente o comportamento e a química cerebral, relacionados à depressão e à ansiedade nos camundongos, fato que se deve à modulação da microbiota intestinal pela administração dos prebióticos.

Outro achado importante do estudo foi a diminuição dos níveis plasmáticos de corticosterona induzida pelo estresse, especialmente diante da combinação entre FOS e GOS. Em conclusão, este estudo demonstra a ação positiva dos prebióticos FOS e GOS na redução de comportamentos depressivos e de ansiedade em ratos.

E, por fim, o estudo de Heym et al. (2019) investigou as relações entre microbiota intestinal, inflamação e fatores de risco psicológico e resiliência para depressão (autojulgamento, identificação e empatia). A pesquisa envolveu quarenta indivíduos adultos, que já faziam parte de um projeto psicométrico maior.

Os participantes preencheram questionários de auto relato para depressão, autojulgamento, identificação e empatia. Também foram coletadas amostras de fezes e de sangue, a fim de avaliar, respectivamente, a composição da microbiota e a presença de moléculas pró-inflamatórias (PCR e IL-6) nos participantes.

Os resultados do estudo demonstraram que a abundância de Lactobacillus spp. se associou ao aumento do autojulgamento positivo e a uma identificação mais baixa, enquanto a PCR foi inversamente associada com a empatia cognitiva. No entanto, Heym et al. (2019), neste estudo, Bifidobacterium spp. e IL-6 não apresentaram relação com nenhuma medida psicométrica. Tais resultados são, possivelmente, inéditos, e por isso o mecanismo dessas associações ainda precisa ser melhormente esclarecido. 


\section{Considerações Finais}

O impacto direto e significativo do intestino e, especificamente, sua microbiota, sobre o sistema nervoso central tem sido consolidado em toda literatura, fato que coloca, cada vez mais, o eixo intestino-cérebro em posição de destaque nos estudos sobre desordens mentais. Assim, todos os estudos avaliados nesta revisão apresentaram resultados positivos em relação a essa tese.

A capacidade da microbiota intestinal em sintetizar uma grande variedade de neurotransmissores e, assim, regular a função neural e a homeostase, é um dos principais mecanismos de ação da microbiota sobre o sistema nervoso, sendo que um desequilíbrio em sua diversidade pode acarretar, dentre outras consequências, em alterações no comportamento, quadros de depressão, ansiedade e estresse, como observado nos estudos acima descritos.

Por fim, ressalta-se a necessidade da realização de mais estudos com essa temática, preferencialmente envolvendo seres humanos, buscando esclarecer os mecanismos da ação da microbiota intestinal sobre o sistema nervoso, incluindo o papel dos probióticos e prebióticos nesse sistema. Ainda, fazem-se necessárias mais pesquisas que avaliem os possíveis benefícios do transplante da microbiota de indivíduos saudáveis para indivíduos com prejuízos na função neurobiológica.

\section{Referências}

Barbosa, FT, Cunha, RM, Pinto, ALCLT. (2008). Delirium pós-operatório em idosos. Rev Bras Anestesiol, 58(6).

Bastiaanssen, TFS, Cussotto, S, Claesson, MJ, Clarke, G, Dinan, TG \& Cryan, JF. (2020). Gutted! Unraveling the Role of the Microbiome in Major Depressive Disorder. Harvard Review of Psychiatry, 28(1), 26-39.

Bourassa, MW, Alim, I, Bultman, SJ \& Ratan, RR. (2016). Butyrate, neuroepigenetics and the gut microbiome: Can a high fiberdiet improve brain health? Neuroscience Letters, 625, 56-63.

Burokas, A, Arboleya, S, Moloney, RD, Peterson, VL, Murphy, K, Clarke, G, Stanton, C, Dinan, TG, Cryan, JF. (2017). Targeting the Microbiota-Gut-Brain Axis: Prebiotics Have 
Anxiolytic and Antidepressantlike Effects and Reverse the Impact of Chronic Stress in Mice. Biological Psychiatry, 82(7), 472-487.

Cerejeira, J, Firmino, H \& Vaz-Serra, A. (2010). The neuroinflammatory hypothesis of delirium. Acta Neuropathol, 119(6), 737-754.

Clarke, G, Grenham, S, Scully, P, Fitzgerald, P, Moloney, RD, Shanahan, F, Dinan, TG, Cryan, JF. (2013). The microbiome-gut-brain axis during early life regulates the hippocampal serotonergic system in a sex-dependent manner. Molecular Psychiatry, 18(6), 666-673.

Cryan, JF, O’Mahony, SM. (2011). The microbiome-gut-brain axis: from bowel to behavior. Neurogastroenterol Motil, 23(3), 187-192.

Del'arco, APWT, Magalhães, P, Quilici, FA. (2017). SIM Brasil study - Women's Gastrointestinal Health: gastrointestinal symptoms and impact on the Brazilian women quality of life. Arq Gastroenterol, 54(2), 115-122.

Dinan, TG, Cryan, JF. (2017). The Microbiome-Gut-Brain Axis in Health and Disease. Gastroenterol Clin North Am., 46(1), 77-89.

Dinan, TG, Stanton, C, Cryan, JF. (2013). Psychobiotics: a novel class of psychotropic. Biol Psychiatry, 74(10), 720-6.

Dinan, TG, Stilling, RM, Stanton, C, Cryan, JF. (2015). Collective unconscious: How gut microbes shape human behavior. Journal of Psychiatric Research, 63, 1-9.

Gareau, MG, Wine, E, Rodrigues, DM, Cho, JH, Whary, MT, Philpott, DJ, Macqueen, G, Sherman, PM. (2011). Bacterial infection causes stress-induced memory dysfunction in mice. Gut, 60(3), 307-17.

Gershon, MD, Tack, J. (2007). The serotonin signaling system: from basic understanding to drug development for functional GI disorders. Gastroenterology, 132(1), 397-414. 
Heym, N, Heasman, BC, Hunter, K, Blanco, SR, Wang, GY, Siegert, R, Cleare, A, Gibson, Kumari, V, Sumich, AL. (2019). The role of microbiota and inflammation in self-judgement and empathy: implications for understanding the brain-gut-microbiome axis in depression. Psychopharmacology, 236(5), 1459-1470.

Kelly, JR, Clarke, G, Cryan, JF, Dinan, TG. (2016). Brain-Gut-Microbiota axis: Challenges for Translation in Psychiatry. Annals of Epidemiology, 26(5), 366-72.

Kelly, JR, Borre, Y, O’Brien, C, Patterson, E, El Aidy, S, Deane, J, Kennedy, PJ, Beers, S, Scott, K, Fitzgerald, P, Ross, P, Stanton, C, Clarke, G, Cryan, JF, Dinan, TG. (2016). Transferring the blues: Depression-associated gut microbiota induces neurobehavioural changes in the rat. Journal of Psychiatric Research, 82, 109-18.

Kelly, JR, Kennedy, PJ, Cryan, JF, Dinan, TG, Clarke, G \& Hyland, NP. (2015). Breaking down the barriers: the gut microbiome, intestinal permeability and stress-related psychiatric disorders. Front Cell Neurosci, 9, 392.

Lach, G, Morais, LH, Costa, APR, Hoeller, AA. (2017). Envolvimento da flora intestinal na modulação de doenças psiquiátricas. Vittalle - Revista de Ciências da Saúde, 29(1), 64-82.

Liu, P, Peng, G, Zhang, N, Wang, B, Luo, B. (2019). Crosstalk Between the Gut Microbiota and the Brain: An Update on Neuroimaging Findings. Front Neurol, 10, 883.

Liu, Q. F., Kim, H. M., Lim, S., Chung, M. J., Lim, C. Y., Koo, B. S., Kang, S. S. (2020). Effect of probiotic administration on gut microbiota and depressive behaviors in mice. DARU J Pharm Sci.

Ministério da Saúde. Biblioteca Virtual em Saúde. (2015). Ansiedade. Retrieved February 04, from http://bvsms.saude.gov.br/dicas-em-saude/470-ansiedade.

Organização Pan-Americana de Saúde. Organização Mundial da Saúde. (2018). Folha informativa - Transtornos mentais. Retrieved February 04, from https://www.paho.org/bra/index.php?option=com_content\&view=article\&id=5652:folhainformativa-transtornos-mentais\&Itemid=839. 
Ozan, ZT, Tanik, N, Inan, LE. (2019). Constipation is associated with tension type headache in women. Arq Neuropsiquiatr, 77(3), 161-165.

Pan, JX, Deng, FL, Zeng, BH, Zheng, P, Liang, WW, Yin, BM, Wu, J, Dong, MX, Luo, Y. Y, Wang, HY, Wei, H \& Xie, P. (2019). Absence of gut microbiota during early life affects anxiolytic Behaviors and monoamine neurotransmitters system in the hippocampal of mice. Journal of the Neurological Sciences, 400, 160-168.

Pereira, AS, Shitsuka, DM, Parreira, FJ \& Shitsuka, R. (2018). Metodologia do trabalho científico. [e-book]. Disponível em:

https://repositorio.ufsm.br/bitstream/handle/1/15824/Lic_Computacao_MetodologiaPesquisa-Cientifica.pdf?sequence=1. Acesso em: 29 ago. 2019.

Reigstad, CS, Salmonson, CE, Rainey, JF, Szurszewski, JH, Linden, DR, Sonnenburg, JL, Farrugia, G \& Kashyap, PC. (2015). Gut microbes promote colonic serotonin production through an effect of short-chain fatty acids on enterochromaffin cells. FASEB J, 29(4), 13951403.

Schmidt, K, Cowen, PJ, Harmer, CJ, Tzortzis, G, Errington, S, Burnet, PWJ. (2015). Prebiotic intake reduces the waking cortisol response and alters emotional bias in healthy volunteers. Psychopharmacology (Berl), 232(10), 1793-801.

Stilling, RM, Dinan, TG, Cryan, JF. (2014). Microbial genes, brain \& behaviour - epigenetic regulation of the gut-brain axis. Genes, Brain and Behavior, 13(1), 69-86.

World Health Organization. (2017). Depression and Other Common Mental Disorders: Global Health Estimates. Retrieved February 04, from https://apps.who.int/iris/bitstream/handle/10665/254610/WHO-MSD-MER-2017.2-eng.pdf.

Xiao, HW, Ge, C, Feng, GX, Li, Y, Luo, D, Dong, JL, Li, H, Wang, H, Cui, M \& Fan, SJ. (2018). Gut microbiota modulates alcohol withdrawal-induced anxiety in mice. Toxicology Letters, 287, 23-30. 
Yano, JM, Yu, K, Donaldson, GP, Shastri, GG, Ann, P, Ma, L, Nagler, CR, Ismagilov, RF, Mazmanian, SK \& Hsiao, EY. (2015). Indigenous Bacteria from the Gut Microbiota Regulate Host Serotonin Biosynthesis. Cell, 161(2), 264-276.

Zhang, J, Bi, JJ, Guo, GJ, Yang, L, Zhu, B, Zhan, GF, Li, S, Huang, NN, Hashimoto, K, Yang, C \& Luo, AL. (2019). Abnormal composition of gut microbiota contributes to delirium-like behaviors after abdominal surgery in mice. CNS Neurosci Ther, 25(6), 685-696.

\title{
Porcentagem de contribuição de cada autor no manuscrito
}

\author{
Ingrid Gabriela de Oliveira Tonini - 60\% \\ Diana Souza Santos Vaz - 10\% \\ Caryna Eurich Mazur - 30\%
}

